\title{
Epigenetic Regulation of Apoptosis and Cell Cycle in Osteosarcoma
}

\author{
Krithi Rao-Bindal ${ }^{1}$ and Eugenie S. Kleinerman ${ }^{1,2}$ \\ ${ }^{1}$ Department of Pediatrics, The University of Texas MD Anderson Cancer Center, Houston, TX 77030, USA \\ ${ }^{2}$ Division of Pediatrics, Unit 87, The University of Texas MD Anderson Cancer Center, 1515 Holcombe Boulevard, Houston, \\ TX 77030, USA
}

Correspondence should be addressed to Eugenie S. Kleinerman, ekleiner@mdanderson.org

Received 10 August 2010; Accepted 18 November 2010

Academic Editor: H. Kovar

Copyright ( $\odot 2011$ K. Rao-Bindal and E. S. Kleinerman. This is an open access article distributed under the Creative Commons Attribution License, which permits unrestricted use, distribution, and reproduction in any medium, provided the original work is properly cited.

\begin{abstract}
The role of genetic mutations in the development of osteosarcoma, such as alterations in p53 and Rb, is well understood. However, the significance of epigenetic mechanisms in the progression of osteosarcoma remains unclear and is increasingly being investigated. Recent evidence suggests that epigenetic alterations such as methylation and histone modifications of genes involved in cell cycle regulation and apoptosis may contribute to the pathogenesis of this tumor. Importantly, understanding the molecular mechanisms of regulation of these pathways may give insight into novel therapeutic strategies for patients with osteosarcoma. This paper serves to summarize the described epigenetic mechanisms in the tumorigenesis of osteosarcoma, specifically those pertaining to apoptosis and cell cycle regulation.
\end{abstract}

\section{Introduction}

Osteosarcoma is the most common primary malignant tumor of the bone in children and adolescents. As a result of recent advances in chemotherapy, long-term survival rates for osteosarcoma patients with no detectable metastases at diagnosis have improved dramatically. However, for patients that present with metastasis or have disease recurrence, the long-term survival rate is less than 20\% [1-3]. Therefore, there is an ongoing need to understand the biology of osteosarcoma progression and metastasis in order to identify new therapeutic approaches.

Numerous studies have investigated the pathogenesis of osteosarcoma. This tumor has generally been associated with alterations in genes involved in cell cycle regulation and apoptosis. Most notably, the p53 and retinoblastoma protein $(\mathrm{Rb})$ pathways have been shown to play a role in the progression of osteosarcoma [4-7]. However, much of the focus has been on understanding point mutations or deletions, disregarding any potential role of epigenetic mechanisms in the inactivation of these and other important pathways.
Epigenetics involves changes in the activation of genes without altering the basic structure of DNA. This includes but is not limited to $\mathrm{CpG}$ island methylation within gene promoter regions and acetylation, deacetylation, and/or methylation of histone proteins $[8,9]$. Epigenetic regulation has been considered a mechanism for the inactivation of tumor suppressor pathways in several types of cancer. These changes can impact gene expression, but how this may contribute to the process of tumorigenesis requires further investigation.

Recent advances in the study of the role of epigenetics in the progression of osteosarcoma have increased the understanding of the pathogenesis of this disease, an area which is complex and not well defined. Altering gene expression and the signaling pathways that control the cell cycle and apoptosis can contribute to the tumorigenic process and cell transformation from a normal to a malignant phenotype. This paper serves to review what is currently known about the effects of aberrant methylation and other epigenetic mechanisms on the regulation of cell cycle and apoptosis in osteosarcoma. 


\section{Rb Pathway}

Retinoblastoma protein $(\mathrm{Rb})$ is a tumor suppressor protein that is inactivated in several types of cancer [10]. It has been shown to play a role in cell cycle control by inhibiting entry into the S-phase, thus creating a G1 checkpoint [11, 12]. About $70 \%$ of human primary osteosarcoma tumors have molecular aberrations in the $\mathrm{Rb}$ gene. The most common alterations include genetic deletions, mutations, and structural rearrangements [1,13-15]. While inactivation by hypermethylation of the $\mathrm{Rb}$ gene has been shown to contribute to pathogenesis of other tumor types such as retinoblastoma [16], analysis of patient samples has suggested that this inactivation may not play an essential role in the progression of osteosarcoma. In one study, only 6 of 76 patients displayed heterozygous $\mathrm{Rb}$ methylation and 6 out of 41 patients displayed $\mathrm{Rb}$ promoter methylation. It is important to note, however, that loss of the Rb gene was only detectable in $37.2 \%$ of these patients, which is considerably lower than previously reported data [17]. Therefore, further analysis of $\mathrm{Rb}$ methylation in osteosarcoma is warranted. It has been shown that Rb-dependent G1 arrest involves p16 ${ }^{\text {INK4A }}$ inhibition of cyclin D/cdk4 and cyclin D/cdk6 complexes, which normally initiate the phosphorylation of $\mathrm{Rb}$ [18]. Therefore, alterations in $\mathrm{Rb}$, cyclin $\mathrm{D}$, cdk4/6, or p $16^{\text {INK4A }}$ may result in a loss of the G1 checkpoint, leading to the accumulation of genetic damage which may contribute to tumor development (Figure 1). Until recently, epigenetic modifications of the $\mathrm{p} 16^{\mathrm{INK} 4 \mathrm{~A}}$ gene, a tumor suppressor that is often altered in osteosarcoma cell lines [19], were not investigated. In a study with p16-negative osteosarcoma samples, 8/15 had total or partial CpG methylation of the p $16^{\mathrm{INK} 4 \mathrm{~A}}$ promoter and $6 / 15$ were pRb-negative [20]. Overall, these data suggest that in addition to other mechanisms of $\mathrm{Rb}$ pathway inhibition, promoter methylation of either $\mathrm{Rb}$ or p16 ${ }^{\mathrm{INK} 4 \mathrm{~A}}$ may play a role in the disruption of cell cycle control, promoting the development of osteosarcoma.

\section{3. p53 Pathway}

The p53 gene (TP53) is known as the most commonly mutated gene in human cancers [1, 21-23]. P53 plays an important role in the regulation of apoptosis, cell cycle arrest, and DNA repair. When DNA damage occurs, p53 upregulates WAF/CIP resulting in increased p21 protein. P21 can then bind to and inhibit G1-S/CDK and S/CDK complexes to arrest cell division. If damage is irreparable, activated p53 can directly regulate the expression of apoptotic genes, resulting in the initiation of apoptosis [23]. The frequency of $\mathrm{p} 53$ alterations in osteosarcoma ranges from $\sim 30 \%$ point mutations to $\sim 80 \%$ allelic loss, suggesting that p53 status plays an important role in the tumorigenesis of osteosarcoma [1]. However, few groups have investigated the role of epigenetic regulation of p53 pathways in osteosarcoma. Recently a novel protein, hypermethylated in cancer (HIC1), was identified to modulate p53-dependent apoptosis (Figure 1). Inactivation of $\mathrm{HICl}$ results in the upregulation of SIRT1 deacetylase which then deacetylates and inactivates p53. This results in the circumvention of

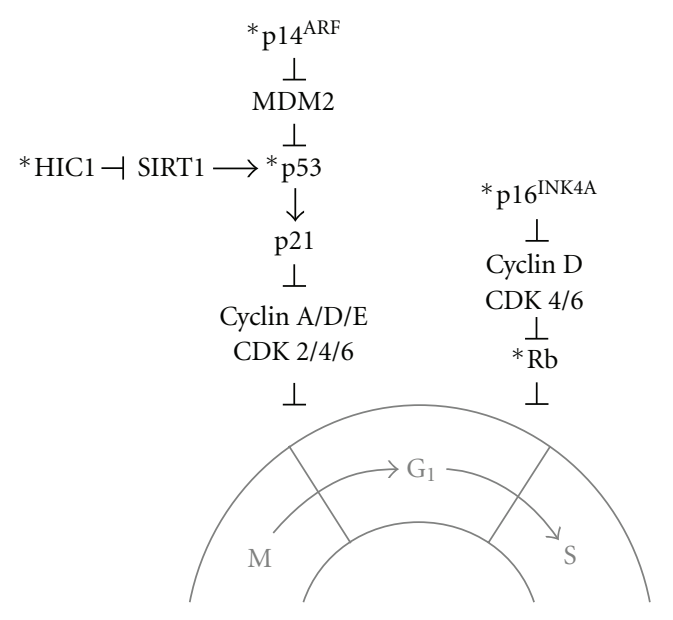

FIGURE 1: Schematic model of epigenetic events that regulate cell cycle progression in osteosarcoma. The cell cycle regulators $\mathrm{Rb}, \mathrm{p} 53$, p16 ${ }^{\mathrm{INK} 4 \mathrm{~A}}, \mathrm{p} 14^{\mathrm{ARF}}$, and $\mathrm{HIC} 1$ have been found to be hypermethylated at the gene promoter in osteosarcoma $(*)$. These alterations may contribute to dysregulation of cell cycle control (loss of the G1 checkpoint) and may promote tumor development.

apoptosis and then cells are able to survive DNA damage, a process that may promote tumor development [24]. In order to investigate the mechanism for loss of function of $\mathrm{HIC1}$ and p53 in osteosarcoma, Chen et al. analyzed the regulation of $\mathrm{HIC1}$ in tumors of $\mathrm{HICl}^{+/-} \mathrm{p} 53^{+/-}$mice. Eight of 13 osteosarcomas demonstrated HIC1 1b promoter hypermethylation and 2/13 had hypermethylation of the HIC1 1a promoter. Both alterations were associated with loss of HIC1 expression, whereas tumors with abundant HIC1 expression had no apparent hypermethylation of the HIC1 promoter. In addition, $2 / 4$ osteosarcomas in $\mathrm{p} 53^{+/-}$mice had abundant HIC1 $1 \mathrm{~b}$ hypermethylation. This suggests that loss of HIC1 function resulting from promoter hypermethylation, along with inactivation of p53, is associated with the development of osteosarcoma. To further examine whether $\mathrm{HIC} 1$ promoter hypermethylation with p53 inactivation was important in the development of human osteosarcomas, Chen et al. analyzed 44 osteosarcoma patient samples. It was demonstrated that $8 / 21(38 \%)$ tumors with p53 mutations and $2 / 23(9 \%)$ without p53 mutations were characterized by HIC1 promoter hypermethylation [25]. In addition, it has been found that $17 \%$ of pediatric osteosarcomas display hypermethylation of the HIC1 promoter [26]. This further validates that in addition to mutation or deletion of the p53 gene, regulation of the p53 pathway by HIC1 promoter hypermethylation resulting in p53 inactivation also plays an important role in the development of osteosarcoma.

\section{4. p14 ${ }^{\mathrm{ARF}} /$ CDKN2A}

The $\mathrm{p} 14^{\mathrm{ARF}}$ protein, which is encoded by the CDKN2A gene, is critical in the regulation of cell cycle control (Figure 1). $\mathrm{P} 14^{\mathrm{ARF}}$ regulates $\mathrm{p} 53$ function by inhibiting MDM2, allowing p53 to upregulate p21 expression. P21 can then bind to and inactivate cyclin/CDK complexes, resulting in G1 arrest [27]. 
In an examination of tissue samples from 59 osteosarcoma patients, it was demonstrated that epigenetic alterations in p $14^{\mathrm{ARF}}$ correlated with poor prognosis. Using methylationspecific polymerase chain reaction (PCR), it was shown that $15 / 32(47 \%)$ osteosarcomas had aberrant methylation of the $\mathrm{p} 14^{\mathrm{ARF}}$ gene (CDKN2A) promoter. As anticipated, these 15 osteosarcomas with methylated p14 ARF showed negative or weak expression of the $\mathrm{p} 14^{\mathrm{ARF}}$ protein. This confirms that methylation of the $\mathrm{p} 14^{\mathrm{ARF}}$ gene promoter is associated with loss of protein expression. Methylation did not correlate with age, gender, location of tumor, tumor volume, stage, histologic subtype, or chemotherapeutic response. Interestingly, the patients with p $14^{\mathrm{ARF}}$ methylation had a lower median survival than the patients without $\mathrm{p} 14^{\mathrm{ARF}}$ methylation, which was statistically significant according to Kaplan-Meier's survival analysis. This suggests that aberrant p14 ${ }^{\mathrm{ARF}}$ promoter methylation correlates with poor survival in patients with osteosarcoma. In addition, 9/14 patients with p14 ${ }^{\mathrm{ARF}}$ promoter methylation developed metastases. Associated deaths correlated with the incidence of metastasis but were not significant due to the size of the cohort [28]. Overall, these data suggest that $\mathrm{p} 14^{\mathrm{ARF}}$ promoter methylation may result in the loss of p53-dependent G1 arrest, which may promote tumor development. However, further study into the significance of $\mathrm{p} 14^{\mathrm{ARF}}$ promoter methylation in primary osteosarcoma and metastasis is warranted and may provide further insight into the importance of $\mathrm{p} 14^{\mathrm{ARF}}$ methylation in osteosarcoma progression.

\section{RASSF1A}

Ras association domain family 1A (RASSF1A) is a newly identified tumor suppressor gene that is involved in pathways regulating cell cycle arrest and apoptosis. Although unclear, RASSF1A has been identified to be involved in death receptor-dependent apoptosis [29]. RASSF1A has been demonstrated to be silenced in cancers of the ovary, nasopharynx, kidney, stomach, prostate, urinary bladder, thyroid, and neuroblastoma [30-37]. The method of RASSF1A silencing in all of these tumor types was determined to be a result of aberrant methylation of the RASSF1A promoter. Recently, RASSF1 was also shown to be a tumor suppressor in osteosarcoma. Lim et al. investigated the expression of RASSF1A in primary osteosarcomas and cell lines and demonstrated a lack of RASSF1A expression in 4/10 primary and 5/6 cell lines. Upon treatment of these RASSF1A-negative cell lines with the demethylating agent 5aza-2' -deoxycytidine, RASFF1A expression was upregulated. This suggests that RASSF1A promoter methylation may be a possible mechanism for the transcriptional silencing of RASSF1A. In contrast with these results, Lim et al. found that several primary osteosarcomas and one cell line (SAOS-2) did not display methylation of the RASSF1A promoter [38]. Therefore, further studies incorporating a greater number of osteosarcoma samples and cell lines are warranted. Additionally, Hou et al. performed a thorough analysis of promoter hypermethylation of a wide of array of genes in osteosarcoma and normal tissues using Q-MSP analysis. RASSF1A promoter hypermethylation was present in $14.29 \%$ (mean) of tumor tissues and in $1.29 \%$ (mean) of normal tissues, demonstrating that promoter methylation is a method of RASSF1A silencing in osteosarcoma [39]. This validates that loss of RASSF1A by promoter methylation may play a role in the development of osteosarcoma by dysregulation of cell cycle control and apoptosis.

\section{Monomethyl Histone H3 Lysine 27}

Several groups have demonstrated the various histone modifications that occur during apoptosis, termed the "apoptotic histone mark" [40]. Histone tails are known to be posttranslationally modified by acetylation, methylation, phosphorylation and ubiquitination of lysine, and/or arginine residues $[41,42]$. One such modification, methylation of lysine 27 in histone $\mathrm{H} 3$, was associated with gene repression and has been implicated in tumorigenesis [43-45]. In osteosarcoma cells, induction of lysine 27 methylation in histone $\mathrm{H} 3$ has been associated with caspase-dependent apoptosis and cell cycle arrest, suggesting that this epigenetic mark may play a possible role in these processes [46]. Conversely, osteosarcoma cells undergoing apoptosis displayed elevated levels of monomethylated histone H3 lysine 27 [47]. These studies are the first to provide evidence that epigenetic modification of histone $\mathrm{H} 3$ by lysine 27 methylation may be linked with apoptosis in osteosarcoma. Although a correlative study, this finding suggests a possible role for histone modifications in the induction of apoptosis in osteosarcoma.

\section{Discussion}

Osteosarcoma is a relatively rare disease, accounting for about $5 \%$ of all pediatric cancers. However, more than $30 \%$ of patients with osteosarcoma die of pulmonary metastasis within 5 years after diagnosis [48]. While adjuvant chemotherapy has improved overall survival rates compared to surgery alone, the fatality rates have remained unchanged for more than 20 years. Therefore, there is an ongoing need for new therapeutic strategies and a more thorough understanding of the genetic and molecular mechanisms that participate in the development of osteosarcoma and in the metastatic process, particularly to the lung. Multiple pathways have been implicated in the pathogenesis of osteosarcoma. Specifically, pathways involving cell cycle and apoptosis have been found to play a role in tumorigenesis. While there is abundant evidence that pathways involving p53, Rb, and many key mediators of cell cycle regulation and apoptosis contribute to osteosarcoma, how these critical genes are altered is not clearly understood. Recent advances in the study of epigenetics have shown that these pathways may be regulated by methylation, histone modifications, and other epigenetic mechanisms. This paper highlights the importance of these epigenetic events in the development of osteosarcoma, specifically pertaining to cell cycle regulation and apoptosis. Overall, a growing body of evidence suggests that use of therapeutic agents that target epigenetic mechanisms may be beneficial for patients with osteosarcoma. 


\section{Acknowledgments}

This work was supported by National Cancer Institute Grant no. CA42992 (E.S.K.) and National Institutes of Health core Grant no. CA16672. The authors would also like to thank the American Legion Auxiliary for the Fellowship Award to Krithi Rao-Bindal.

\section{References}

[1] L. L. Wang, "Biology of osteogenic sarcoma," Cancer Journal, vol. 11, no. 4, pp. 294-305, 2005.

[2] W. S. Ferguson and A. M. Goorin, "Current treatment of osteosarcoma," Cancer Investigation, vol. 19, no. 3, pp. 292$315,2001$.

[3] N. Marina, M. Gebhardt, L. Teot, and R. Gorlick, "Biology and therapeutic advances for pediatric osteosarcoma," Oncologist, vol. 9, no. 4, pp. 422-441, 2004.

[4] S. D. Berman, E. Calo, A. S. Landman et al., "Metastatic osteosarcoma induced by inactivation of $\mathrm{Rb}$ and $\mathrm{p} 53$ in the osteoblast lineage," Proceedings of the National Academy of Sciences of the United States of America, vol. 105, no. 33, pp. 11851-11856, 2008.

[5] J. F. McIntyre, B. Smith-Sorensen, S. H. Friend et al., "Germline mutations of the p53 tumor suppressor gene in children with osteosarcoma," Journal of Clinical Oncology, vol. 12, no. 5, pp. 925-930, 1994.

[6] C. W. Miller, A. Aslo, C. Tsay et al., "Frequency and structure of p53 rearrangements in human osteosarcoma," Cancer Research, vol. 50, no. 24, pp. 7950-7954, 1990.

[7] C. R. Walkley, R. Qudsi, V. G. Sankaran et al., "Conditional mouse osteosarcoma, dependent on p53 loss and potentiated by loss of Rb, mimics the human disease," Genes and Development, vol. 22, no. 12, pp. 1662-1676, 2008.

[8] L. Ellis, P. W. Atadja, and R. W. Johnstone, "Epigenetics in cancer: targeting chromatin modifications," Molecular Cancer Therapeutics, vol. 8, no. 6, pp. 1409-1420, 2009.

[9] P. A. Jones and S. B. Baylin, "The epigenomics of cancer," Cell, vol. 128, no. 4, pp. 683-692, 2007.

[10] A. L. Murphree and W. F. Benedict, "Retinoblastoma: clues to human oncogenesis," Science, vol. 223, no. 4640, pp. 10281033, 1984.

[11] M. Hatakeyama and R. A. Weinberg, "The role of RB in cell cycle control," Progress in cell cycle research, vol. 1, pp. 9-19, 1995.

[12] C. Giacinti and A. Giordano, "RB and cell cycle progression," Oncogene, vol. 25, no. 38, pp. 5220-5227, 2006.

[13] R. B. Scholz, H. Kabisch, B. Weber, K. Roser, G. Delling, and K. Winkler, "Studies of the RB1 gene and the p53 gene in human osteosarcomas," Pediatric Hematology and Oncology, vol. 9, no. 2, pp. 125-137, 1992.

[14] B. I. Wadayama, J. Toguchida, T. Shimizu et al., "Mutation spectrum of the retinoblastoma gene in osteosarcomas," Cancer Research, vol. 54, no. 11, pp. 3042-3048, 1994.

[15] N. Araki, A. Uchida, T. Kimura et al., "Involvement of the retinoblastoma gene in primary osteosarcomas and other bone and soft-tissue tumors," Clinical Orthopaedics and Related Research, no. 270, pp. 271-277, 1991.

[16] T. Sakai, J. Toguchida, N. Ohtani, D. W. Yandell, J. M. Rapaport, and T. P. Dryja, "Allele-specific hypermethylation of the retinoblastoma tumor-suppressor gene," American Journal of Human Genetics, vol. 48, no. 5, pp. 880-888, 1991.
[17] A. Patiño-García, E. Sotillo Piñeiro, M. Zalacaín Díez, L. Gárate Iturriagagoitia, F. Antillón Klüssmann, and L. Sierrasesúmaga Ariznabarreta, "Genetic and epigenetic alterations of the cell cycle regulators and tumor suppressor genes in pediatric osteosarcomas," Journal of Pediatric Hematology/Oncology, vol. 25, no. 5, pp. 362-367, 2003.

[18] N. Ohtani, K. Yamakoshi, A. Takahashi, and E. Hara, "The p16-RB pathway: molecular link between cellular senescence and tumor suppression," Journal of Medical Investigation, vol. 51, no. 3-4, pp. 146-153, 2004.

[19] C. W. Miller, A. Aslo, M. J. Campbell, N. Kawamata, B. C. Lampkin, and H. P. Koeffler, "Alterations of the p15, p16, and p18 genes in osteosarcoma," Cancer Genetics and Cytogenetics, vol. 86, no. 2, pp. 136-142, 1996.

[20] M. Serena Benassi, L. Molendini, G. Gamberi et al., "Alteration of prb/p16/cdk4 regulation in human osteosarcoma," International Journal of Cancer, vol. 84, no. 5, pp. 489-493, 1999.

[21] M. Hollstein, D. Sidransky, B. Vogelstein, and C. C. Harris, "p53 Mutations in human cancers," Science, vol. 253, no. 5015, pp. 49-53, 1991.

[22] W. P. Bennett, M. C. Hollstein, I. C. Hsu et al., "Mutational spectra and immunohistochemical analyses of p53 in human cancers," Chest, vol. 101, no. 3, supplement, pp. 19S-20S, 1992.

[23] A. J. Levine, J. Momand, and C. A. Finlay, "The p53 tumour suppressor gene,” Nature, vol. 351, no. 6326, pp. 453-456, 1991.

[24] Y. C. Wen, D. H. Wang, C. Y. RayWhay, J. Luo, W. Gu, and S. B. Baylin, "Tumor suppressor HIC1 directly regulates SIRT1 to modulate p53-dependent DNA-damage responses," Cell, vol. 123, no. 3, pp. 437-448, 2005.

[25] W. Chen, T. K. Cooper, C. A. Zahnow et al., "Epigenetic and genetic loss of Hicl function accentuates the role of p53 in tumorigenesis," Cancer Cell, vol. 6, no. 4, pp. 387-398, 2004.

[26] A. Rathi, A. K. Virmani, K. Harada et al., "Aberrant methylation of the HIC1 promoter is a frequent event in specific pediatric neoplasms," Clinical Cancer Research, vol. 9, no. 10 I, pp. 3674-3678, 2003.

[27] T. Waldman, K. W. Kinzler, and B. Vogelstein, "p21 Is necessary for the p53-mediated $G$ arrest in human cancer cells," Cancer Research, vol. 55, no. 22, pp. 5187-5190, 1995.

[28] J. H. Oh, H. S. Kim, H. H. Kim, W. H. Kim, and S. H. Lee, "Aberrant methylation of p14 gene correlates with poor survival in osteosarcoma," Clinical Orthopaedics and Related Research, no. 442, pp. 216-222, 2006.

[29] S. Baksh, S. Tommasi, S. Fenton et al., "The tumor suppressor RASSF1A and MAP-1 link death receptor signaling to bax conformational change and cell death," Molecular Cell, vol. 18, no. 6, pp. 637-650, 2005.

[30] A. Agathanggelou, S. Honorio, D. P. Macartney et al., "Methylation associated inactivation of RASSF1A from region 3p21.3 in lung, breast and ovarian tumours," Oncogene, vol. 20, no. 12, pp. 1509-1518, 2001.

[31] K. W. Lo, J. Kwong, A. B. Y. Hui et al., "High frequency of promoter hypermethylation of RASSF1A in nasopharyngeal carcinoma," Cancer Research, vol. 61, no. 10, pp. 3877-3881, 2001.

[32] C. Morrissey, A. Martinez, M. Zatyka et al., "Epigenetic inactivation of the RASSF1A 3p21.3 tumor suppressor gene in both clear cell and papillary renal cell carcinoma," Cancer Research, vol. 61, no. 19, pp. 7277-7281, 2001.

[33] D. S. Byun, M. G. Lee, K. S. Chae, B. G. Ryu, and S. G. Chi, "Frequent epigenetic inactivation of RASSF1A by aberrant 
promoter hypermethylation in human gastric adenocarcinoma," Cancer Research, vol. 61, no. 19, pp. 7034-7038, 2001.

[34] M. G. Lee, H. Y. Kim, D. S. Byun et al., "Frequent epigenetic inactivation of RASSF1A in human bladder carcinoma," Cancer Research, vol. 61, no. 18, pp. 6688-6692, 2001.

[35] I. Kuzmin, J. W. Gillespie, A. Protopopov et al., "The RASSF1A tumor suppressor gene is inactivated in prostate tumors and suppresses growth of prostate carcinoma cells," Cancer Research, vol. 62, no. 12, pp. 3498-3502, 2002.

[36] U. Schagdarsurengin, O. Gimm, C. Hoang-Vu, H. Dralle, G. P. Pfeifer, and R. Dammann, "Frequent epigenetic silencing of the CpG island promoter of RASSF1A in thyroid carcinoma," Cancer Research, vol. 62, no. 13, pp. 3698-3701, 2002.

[37] D. Astuti, A. Agathanggelou, S. Honorio et al., "RASSF1A promoter region $\mathrm{CpG}$ island hypermethylation in phaeochromocytomas and neuroblastoma tumours," Oncogene, vol. 20, no. 51, pp. 7573-7577, 2001.

[38] S. Lim, M. H. Yang, J. H. Park et al., "Inactivation of the RASSF1A in osteosarcoma," Oncology reports, vol. 10, no. 4, pp. 897-901, 2003.

[39] P. Hou, M. Ji, B. Yang et al., "Quantitative analysis of promoter hypermethylation in multiple genes in osteosarcoma," Cancer, vol. 106, no. 7, pp. 1602-1609, 2006.

[40] J. Füllgrabe, N. Hajji, and B. Joseph, "Cracking the death code: apoptosis-related histone modifications," Cell Death and Differentiation, vol. 17, pp. 1238-1243, 2010.

[41] B. D. Strahl and C. D. Allis, "The language of covalent histone modifications," Nature, vol. 403, no. 6765, pp. 41-45, 2000.

[42] S. L. Berger, "Histone modifications in transcriptional regulation," Current Opinion in Genetics and Development, vol. 12, no. 2, pp. 142-148, 2002.

[43] A. J. Bannister, R. Schneider, and T. Kouzarides, "Histone methylation: dynamic or static?" Cell, vol. 109, no. 7, pp. 801$806,2002$.

[44] W. Fischle, Y. Wang, and C. D. Allis, "Histone and chromatin cross-talk," Current Opinion in Cell Biology, vol. 15, no. 2, pp. 172-183, 2003.

[45] A. Kirmizis, S. M. Bartley, A. Kuzmichev et al., "Silencing of human polycomb target genes is associated with methylation of histone H3 Lys 27," Genes and Development, vol. 18, no. 13, pp. 1592-1605, 2004.

[46] F. Zhao, Y. Chen, R. Li, Y. Liu, LU. Wen, and C. Zhang, "Triptolide alters histone H3K9 and H3K27 methylation state and induces G0/G1 arrest and caspase-dependent apoptosis in multiple myeloma in vitro," Toxicology, vol. 267, no. 1-3, pp. 70-79, 2010.

[47] M. F. Cheng, C. H. Lee, K. T. Hsia, G. S. Huang, and H. S. Lee, "Methylation of histone $\mathrm{H} 3$ lysine 27 associated with apoptosis in osteosarcoma cells induced by staurosporine," Histology and histopathology, vol. 24, no. 9, pp. 1105-1111, 2009.

[48] A. C. Shor, E. A. Keschman, F. Y. Lee et al., "Dasatinib inhibits migration and invasion in diverse human sarcoma cell lines and induces apoptosis in bone sarcoma cells dependent on Src kinase for survival," Cancer Research, vol. 67, no. 6, pp. 2800$2808,2007$. 


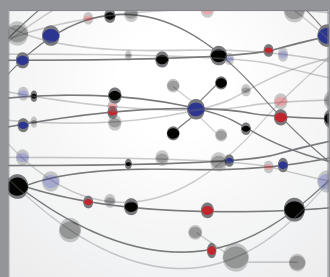

The Scientific World Journal
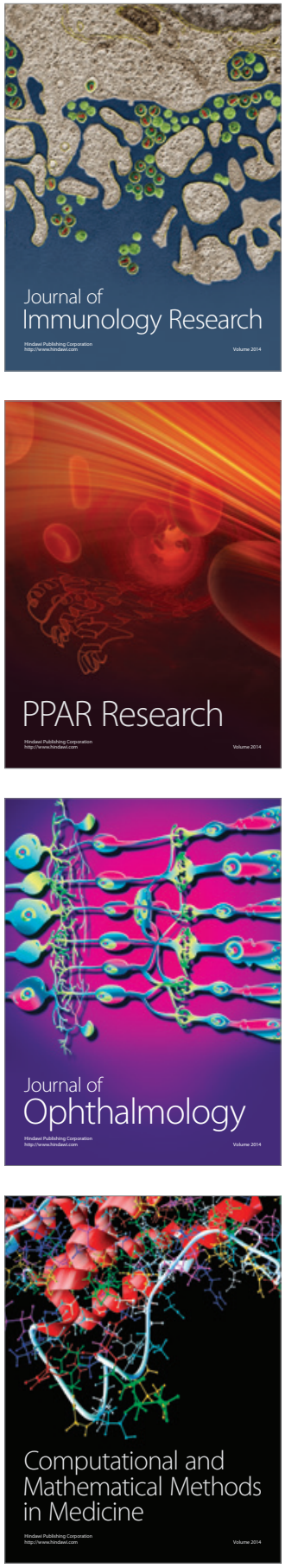

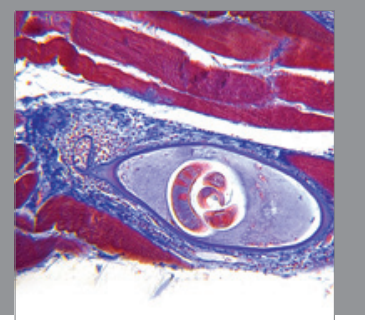

Gastroenterology

Research and Practice
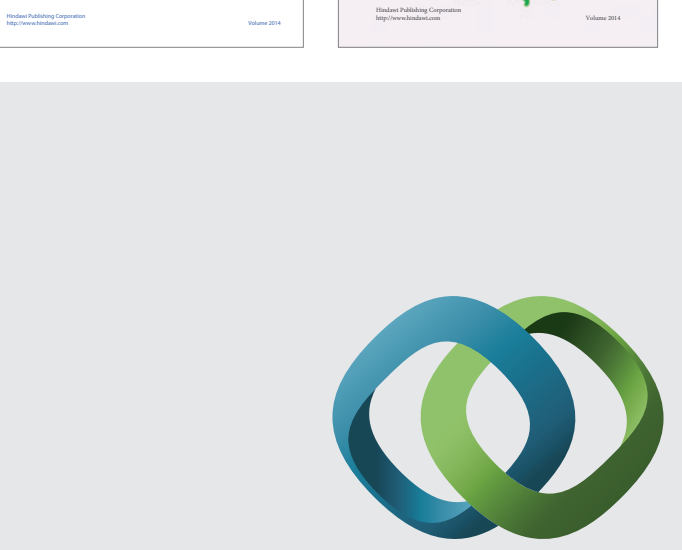

\section{Hindawi}

Submit your manuscripts at

http://www.hindawi.com
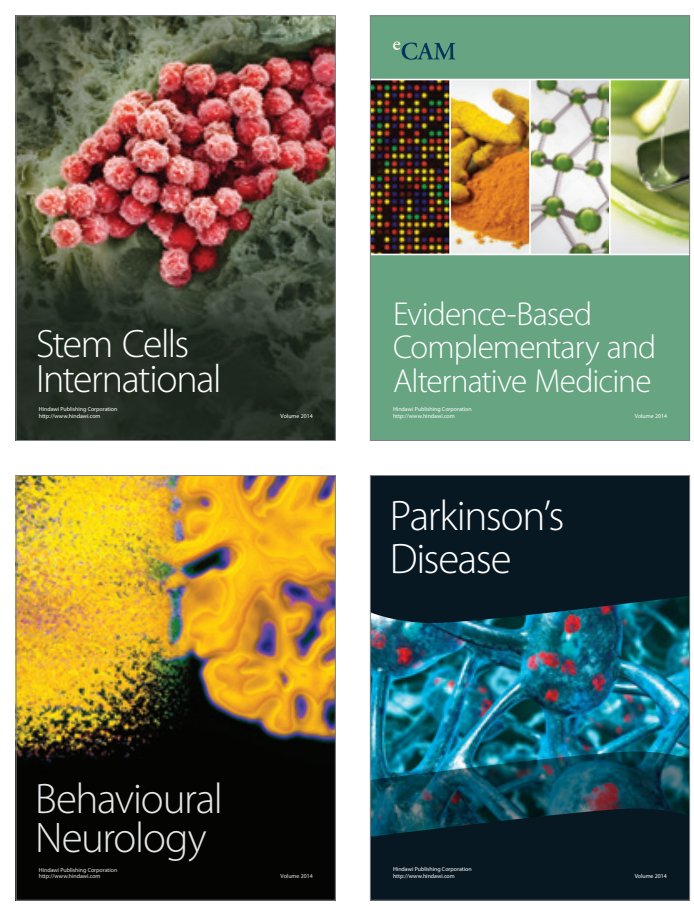

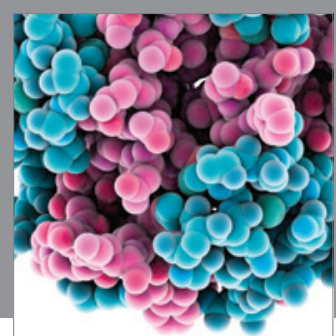

Journal of
Diabetes Research

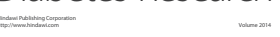

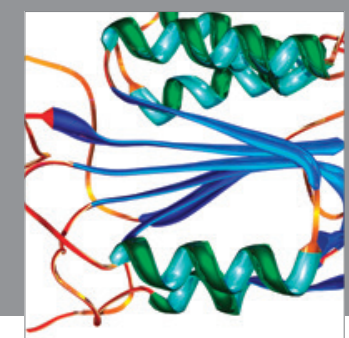

Disease Markers
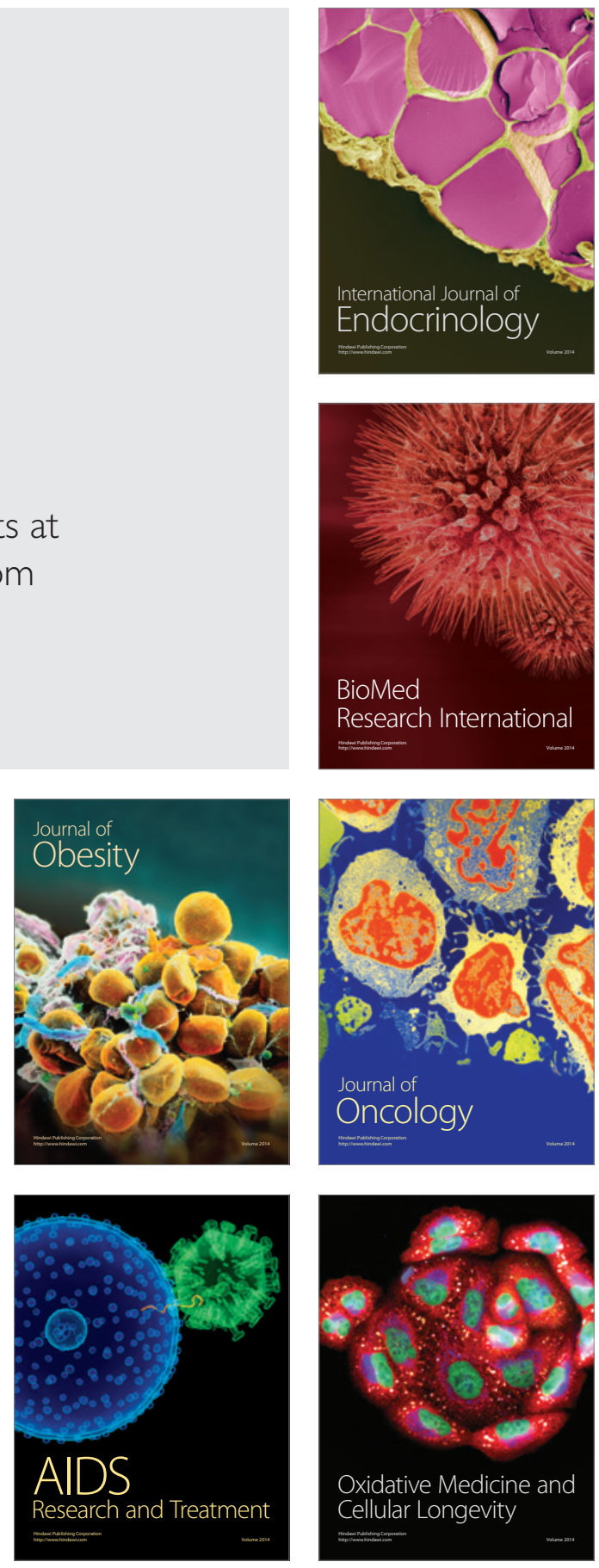